MATEC Web of Conferences 1, 01004 (2012)

DOI: $10.1051 /$ matecconf $/ 20120101004$

(C) Owned by the authors, published by EDP Sciences, 2012

\title{
Nonlinear Modeling and Analysis of a Vertical Springless Energy Harvester
}

\author{
Mohamed Bendame ${ }^{1, a}$ and Eihab Abdel-Rahman ${ }^{1}$ \\ University of Waterloo, 200 University Avenue, Waterloo, Ontario, Canada
}

\begin{abstract}
Harvesting energy from ambient sources has attracted the attention of researchers and scientists over the last few decades. While solar, thermal and wind energies have been exploited over the years, a new type of energy that has emerged in recent years, and is the subject of many research projects, is vibration energy harvesting. In this paper we will describe and analyze a recently proposed vibration energy harvester, namely the "Springless" vibration energy harvester. In this study, we will model and analyze the "Springless" vibration energy harvester in the vertical configuration. The vertically-aligned configuration is used when vibrations are predominantly in the vertical direction. Test results of a prototype model as well as results form a mathematical model describing the behavior of the harvester are presented. Test results show that the "Springless" energy vibration harvester behaves as a softening nonlinear oscillator for excitations above $0.2 g$ with its center frequency shifting to the right. Similar results were obtained using a mathematical model of the underlying impact oscillator.
\end{abstract}

\section{Introduction}

Energy harvesting is the process by which energy is extracted from ambient sources and converted into electrical energy [1][2]. While ambient energy sources such as solar, thermal and wind have been used extensively in a variety of applications, another source of energy that has always attracted attention is vibration energy. While the idea of converting motion into electrical power has been used in kinetic watches for a long time, advances in micro-electronics and low power consumption of silicon-based electronics have given it an added significance. A range of wireless devices with different amplitudes of power consumption and typical battery life are shown in Table 1 [11].

Table 1. Selected battery-operated systems.

\begin{tabular}{l|l|l}
\hline \hline Device Type & Power Consumption & Duration \\
\hline \hline Smartphone & $1 \mathrm{~W}$ & $5 \mathrm{~h}$ \\
\hline MP3 Player & $50 \mathrm{~mW}$ & $15 \mathrm{~h}$ \\
\hline Hearing aid & $1 \mathrm{~mW}$ & 5 days \\
\hline Wireless sensor & $100 \mu W$ & Lifetime \\
\hline Cardiac pacemaker & $50 \mu W$ & 7 years \\
\hline Quartz watch & $5 \mu \mathrm{W}$ & 5 years \\
\hline \hline
\end{tabular}

Due to low-power consumption of these devices, vibration based energy harvesters (VEH) have become an attractive replacements for batteries. VEHs use mechanical oscillators and transducers to collect kinetic energy from ambient vibrations and transform it into electrical power [1][4]. Over the last few years, a wide range of VEHs have been proposed and demonstrated. A single degree-of-freedom spring-mass-damper

\footnotetext{
a e-mail: mbendame@uwaterloo.ca
}

representation of a typical VEH is shown in Figure 1. The seismic mass of the VEH moves under the influence of base excitation supported by a spring. The oscillator attains maximum velocity, and thus input kinetic energy, in a frequency band around its natural frequency defined as: $\omega=\sqrt{\frac{k}{m}}$, where $k$ is the linear stiffness of the spring and $m$ is the effective mass of the oscillator.

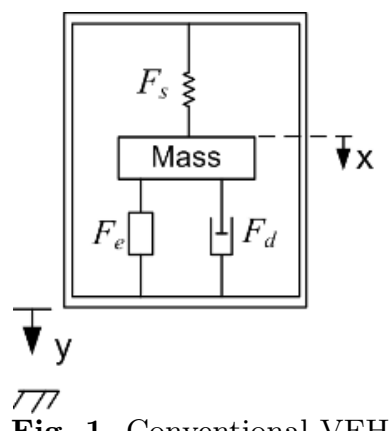

Fig. 1. Conventional VEH

While these types of energy harvesters are capable of generating electrical energy with output power on the order of milli-Watts $[1,7]$, their natural frequency must be tuned to match the frequency of ambient vibrations. These types of harvesters are, by definition, designed to to work at a single frequency. A high Qresonance means very limited practical bandwidths over which energy can be harvested [5]. In environments where ambient vibrations are only available at low frequencies, conventional harvesters prove to be ineffective because of their high center frequencies. In many built environments, ambient vibrations are only available at low frequencies. It is therefore impractical to use traditional VEHs, that usually have 
relatively high center frequency $(\geq 10 \mathrm{~Hz})$, to harvest low frequency $(<10 \mathrm{~Hz})$ vibrations. As a result, there has been an interest in realizing low center frequency VEHs. For example, Fondevilla et al. [8] presented an electromagnetic $\mathrm{VEH}$ designed to harvest random and low frequency sea wave motions.

To overcome the limitation of harvesting from low frequency vibrations a number of approaches have been proposed. Adaptive kinetic energy harvesters, for instance, use mechanisms to adjust or tune the resonant frequency of the harvester so that it matches the frequency of the ambient vibration at all times or widen its bandwidth. Resonant frequency tuning can be achieved by changing the mechanical parameters of the structure while widening the bandwidth can be achieved by employing an array of structures with different resonant frequencies [5].

While these methods can overcome the above mentioned limitations their implementation can be challenging. On one hand, changing the mechanical characteristics of the structure requires replacing parts such as mass and spring. On the other hand, using an array of resonators will increase the size of the harvester, thus making it an unsuitable power source of for portable devices.

In this paper, we analyze a recently proposed lowfrequency harvester, namely the "springless" VEH [6]. Specifically, we study and analyze the response of the vertically aligned configuration of the "springless" electromagnetic VEH experimentally and analytically.

\section{VEH Model}

The springless VEH consists of an electromagnetic transducer, an inertial mass comprised of magnets and a cage, two end limiters consisting of two springs at each end of the housing unit. The carriage moves along the linear guide carrying the assembly with respect to a stationary surface coil in response to base excitations, as shown in Figure 2. The motion of the magnetic carriage induces a voltage $V$ across the coil terminals and proportional to the time rate of change of the magnetic flux within the coil [5];

$$
V=\frac{d \phi}{d x} \frac{d x}{d t}
$$

where $\phi$ is the total magnetic flux and $x$ is the displacement of the magnetic field (carriage) with respect to the coil. The vertical implementation of the VEH, Figure 2, is suitable for environments where motions are predominantly in the vertical direction. The linear guide in this case is aligned with the direction of gravity. It allows the carriage to move along the rail while baring motion in other directions [6]. The carriage carries the inertial mass and the transducer along the guide. The equation of motion of a vertically-aligned harvester is

$$
m \ddot{x}+\left(b_{e}+b_{m}\right) \dot{x}+F(x)=-m \ddot{y}-m g
$$

where $x$ and $y$ are the displacements of the seismic mass $m$ and the frame, respectively, and $F(x)$ is a

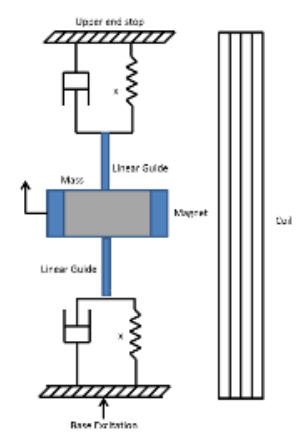

Fig. 2. Vertical Implementation of the VEH

nonlinear-piecewise function that represents the restoring force of the springs. The $\mathrm{VEH}$ harvests kinetic energy transmitted to it from the host vibrations represented by the base acceleration

$$
\ddot{y}=A_{\circ} \cos \Omega t
$$

where $A_{\circ}$ and $\Omega$ are the amplitude and frequency of the external excitation. Two identical springs are used

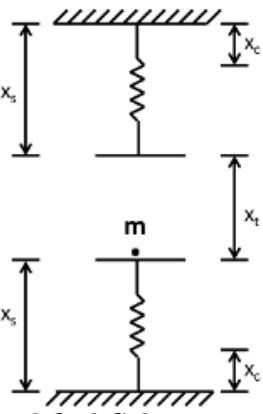

Fig. 3. Simplified Schematic of the VEH

as limiters on either end of the linear guide. The origin of the coordinate system used to describe the seismic mass is placed at the position where it just touches the uncompressed lower spring. The seismic mass $m$ is assumed to be a point mass, as shown in Figure 3. The free distance along the rail (not occupied by the cage) between the upper and lower uncompressed springs is denoted $x_{t}$. The uncompressed length of each spring is denoted $x_{s}$ and the fully compressed length is denoted $x_{c}$. The restoring force, $\mathrm{F}(\mathrm{x})$, is represented by

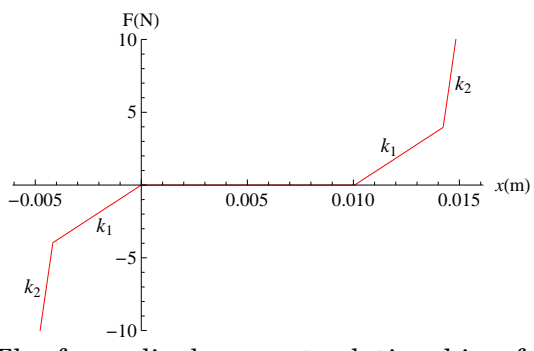

Fig. 4. The force-displacement relationship of the spring 
a piecewise nonlinear function in terms of linear and nonlinear stiffness of the springs and is written as follows:

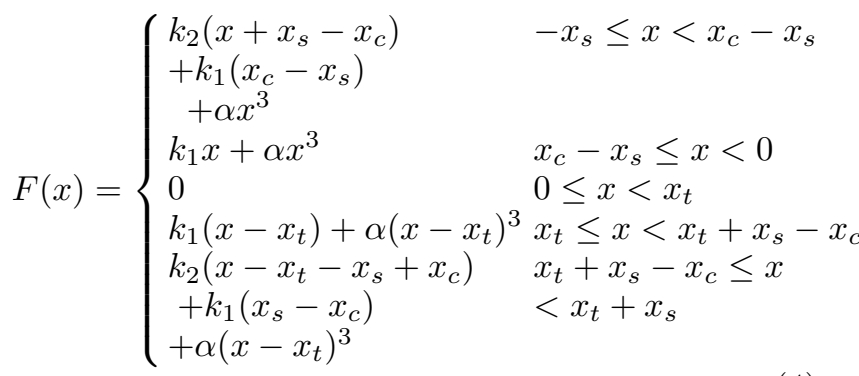

where $k_{1}$ is the linear spring stiffness, $k_{2}>>k_{1}$ is the stiffness of the fully compressed spring, and $\alpha$ is the spring coefficient of cubic nonlinearity. The forcedisplacement relationship is shown in Figure 4. The cubic terms in the equation (4) correspond to the case when the seismic mass fully compresses the springs, thus changing the stiffness to that of a very hard spring.

Three distinct motion regimes can be identified for the vertically-aligned $\mathrm{VEH}$ :

(1) For small excitations (motions where $x_{c}-x_{s}<$ $x \leq 0)$ : the seismic mass will be in contact with the lower spring throughout motion and the system will behave as a linear oscillator with a linear stiffness $k_{1}$.

(2) For moderate excitations (motions where $x<x_{t}$ ): The amplitude of oscillation will be large enough for the seismic mass $m$ to separate from the lower spring without reaching the upper spring. In this region the system behaves like a bouncing ball.

(3) For large excitations (motions where $-x_{s} \leq x \leq$ $\left.x_{s}+x_{t}\right)$ : Motions will be large enough for the seismic mass to leave the lower spring, fly freely along the rail, makes contact the upper spring before descending again.

\subsection{Electromagnetic Damping}

The current passing through the coil creates a magnetic field that opposes the field produced by the magnets. The interaction between the two fields produces a force which opposes the motions of the inertial mass. It is this interaction force that acts as electromagnetic damping, and it can be expressed as [6]

$$
F_{e m}=b_{e} \frac{d x}{d t}
$$

The electrical power is extracted from the mechanical oscillator and is given by [5]

$$
P_{e m}=F_{e m} \frac{d x}{d t}
$$

This power is dissipated in the coil resistance $R_{c}$ and the load resistance $R_{L}$. Equating the power dissipated to that generated by the electromagnetic force gives

$$
P_{e m}=b_{e}\left(\frac{d x}{d t}\right)^{2}=\frac{V^{2}}{R_{L}+R_{C}+j \omega L}
$$

where $L$ is the coil inductance. Substituting for the voltage using Equation (1), we can write the electromagnetic damping as

$$
b_{e}=\frac{1}{R_{L}+R_{C}+j \omega L}\left(\frac{d \Phi}{d x}\right)^{2}
$$

Assuming that the coil inductance is negligible and the magnetic field intensity $B$ is constant, the electromagnetic damping coefficient can be expressed as:

$$
b_{e}=\frac{(B l)^{2}}{R_{L}+R_{C}}
$$

where $l$ is the effective length of the coil. The electrical damping can, therefore, be calculated using Equation (9) and the parameter values given in Table 2.

Table 2. Electromagnetic Transducer Parameters

\begin{tabular}{l|l}
\hline \hline Parameter & Value \\
\hline \hline Magnetic Field B $(\mathrm{T})$ & 0.37 \\
\hline Effective Coil Length l $(\mathrm{m})$ & 1.74 \\
\hline Load Resistance $R_{L}(\Omega)$ & $\infty($ open-loop) \\
\hline Coil Resistance $R_{C}(\Omega)$ & 8.4 \\
\hline \hline
\end{tabular}

\subsection{Mechanical Damping}

The frequency-response curve of the open-loop harvester is used to determine the quality factor $Q$ of the $\mathrm{VEH}$, defined as:

$$
Q_{m}=\frac{f_{0}}{\Delta f}
$$

where $f_{0}$ is the center frequency and $\Delta f=f_{2}-f_{1}$, with $f_{1}$ and $f_{2}$ are the two half-power frequencies. The quality factor for the open-loop harvester $Q_{m}$ can then be used to calculate the mechanical damping of the harvester from the following formula;

$$
Q_{m}=\frac{\sqrt{m k_{1}}}{b_{m}}
$$

where $b_{m}$ is the mechanical damping coefficient of the open-loop harvester. We can find the mechanical damping using Equations (10) and (11) and the values of the systems parameters given in Table 3 as $b_{m}=1.12 \mathrm{~kg} / \mathrm{s}$. The center frequency and half-power bandwidth were found from a frequency-sweep curve of the base acceleration of the VEH at an amplitude of $A_{\circ}=0.1 \mathrm{~g}$ shown in Figure 5 . The total damping of the $\mathrm{VEH}$ is sum of mechanical damping and electromagnetic damping: $b=b_{e}+b_{m}$.

\section{Results}

In this section we will look at experimental test results of the VEH to determine its mechanical and electrical damping coefficients, its center frequency and frequency bandwidth. We will also develop and analyze 
Table 3. VEH Parameters

\begin{tabular}{l|l}
\hline \hline Parameter & Value \\
\hline \hline Mass $\mathrm{m}(\mathrm{kg})$ & 0.095 \\
\hline Stiffness $k_{1}(\mathrm{~N} / \mathrm{m})$ & 700 \\
\hline Center Frequency $f_{0}(\mathrm{~Hz})$ & 18.51 \\
\hline Upper $3 d B$ Frequency $f_{2}(\mathrm{~Hz})$ & 21.35 \\
\hline Lower $3 d B$ Frequency $f_{1}(\mathrm{~Hz})$ & 19.55 \\
\hline \hline
\end{tabular}

a mathematical model using the shooting method (see section 4) to find the periodic orbits of the $\mathrm{VEH}$ and to determine stabilty using Floquet theory. We will apply the shooting method as described in [9].

The experimental tests were carried out on the VEH by mounting it on an electromagnetic shaker. A base acceleration was applied as input excitation. The RMS of the open-loop voltage between the coil terminals was measured using a data acquisition system. The VEH was tested by applying a frequency-sweep of $\Omega$ in the domain of interest while holding the amplitude of base acceleration constant $A_{\circ}$.

\subsection{Experimental Test Results}

\subsubsection{Small Excitations $\left(A_{o} \leq 0.05 \mathrm{~g}\right)$}

Figure 5 shows the experimentally obtained frequencyresponse curves of the RMS voltage for up- and downsweeps of the base acceleration at amplitudes of $A_{\circ}=$ $0.042 \mathrm{~g}$ (green), $0.045 \mathrm{~g}$ (red).

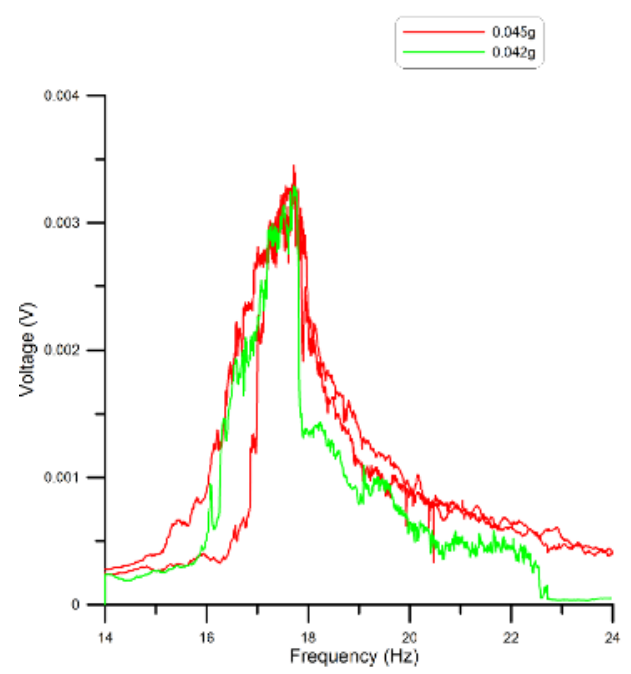

Fig. 5. The frequency-response of the coil RMS voltage under low excitations

In this region the $\mathrm{VEH}$ is expected to behave like a linear oscillator, instead we noticed that it exhibited a hardening effective nonlinearity as indicated by the curves bending to the right and the nonlinear resonance peak shifting to higher frequencies as the excitation amplitude is decreased as indicated by Figure 5 showing the VEH frequency response curves ( $A_{\circ}=0.04 \mathrm{~g}$ (blue), $0.042 \mathrm{~g}$ (green), $0.045 \mathrm{~g}$ (red), and $0.02 \mathrm{~g}$ (blue)). The other noticeable feature is the sudden drop in the amplitude around $\Omega=18 \mathrm{~Hz}$, this is a clear evidence for the coexistence of two branches (upper and lower) of response, jumps between these branches, and hysteresis [9] in the VEH response. The maximum output voltage of the $\mathrm{VEH}$ at the low amplitudes is about $V=4 \mathrm{mV}$ at a resonance frequency $\Omega=18 \mathrm{~Hz}$. Figure 5 shows the experimentally obtained frequency-response curves of the RMS voltage for up- and down- sweeps of the base acceleration at amplitudes of $A_{\circ}=0.04 \mathrm{~g}$ (blue), $0.042 \mathrm{~g}$ (green), $0.045 \mathrm{~g}$ (red), and $0.02 \mathrm{~g}$ (blue).

\subsubsection{Moderate Excitations $\left(0.05 \mathrm{~g}<A_{o}<0.08 \mathrm{~g}\right)$}

For base acceleration amplitudes greater than $0.05 \mathrm{~g}$ and less $0.08 \mathrm{~g}$, the seismic mass separates from the lower spring to travel along the linear guide without touching the upper spring. The experimental frequencyresponse curve of the coil RMS voltage is shown in Figure 6 for an intermediate acceleration amplitudes between $A_{\circ}=0.05-0.08 \mathrm{~g}$. The figure presents an up-sweep (solid line) and a down-sweep (dotted line) of the excitation frequency in the interval $[14-24] \mathrm{Hz}$.

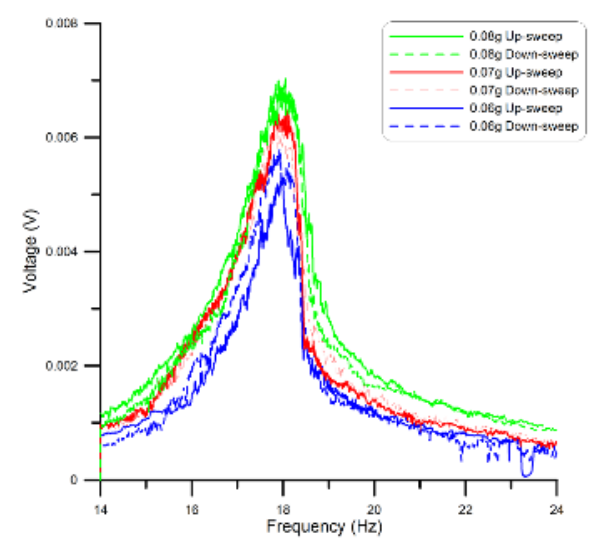

Fig. 6. The frequency-response of the coil RMS voltage under moderate acceleration

Clear evidence again emerges in the results for the coexistence of two branches (upper and lower) of response, and sudden drop in amplitude that indicates jumps between these branches, and hysteresis [9] in the $\mathrm{VEH}$ response. At these moderate amplitude the maximum output voltage of the $\mathrm{VEH}$ was recorder to be around $V=8 \mathrm{~V}$.

\subsubsection{Large Excitations $\left(A_{o}>0.1 \mathrm{~g}\right)$}

The base acceleration amplitudes were set to $A_{\circ}=$ $0.2 \mathrm{~g}, 0.3 \mathrm{~g}, 0.4 \mathrm{~g}$, and $0.5 \mathrm{~g}$ to test the $\mathrm{VEH}$ response for large excitations (motion regime 3). Up-sweep of the frequency of excitation were performed in the interval $14 \mathrm{~Hz}$ to $24 \mathrm{~Hz}$ and the RMS of the voltage across the coil was measured experimentally. The resulting frequency-response curves are shown in Figure 7 in solid lines for the frequency up-sweeps and dashed lines for the frequency down-sweeps. 


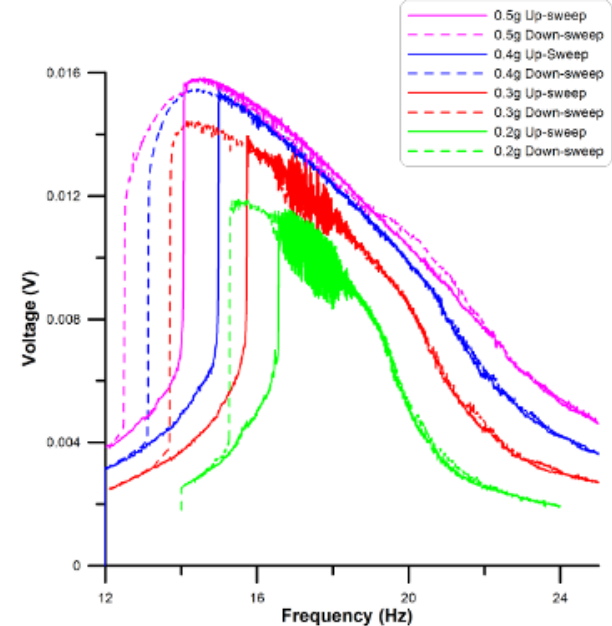

Fig. 7. The frequency-response of the coil RMS voltage under large excitations

At high amplitudes of acceleration we notice that all frequency-response curves are bent to the left indicating a softening type-nonlinearity. As in the case of moderate excitations, jumps between an upper and a lower branches of response and hysteresis between the up- and down-sweeps are observed. The hysteretic region in the frequency spectrum grows to cover more than $1 \mathrm{~Hz}$ and shifts to lower values along the frequency spectrum as the acceleration amplitude grows. Additional features are also observed in the VEH response within this motion regime. The peak voltage is effectively capped to approximately $16 \mathrm{mV}$ at $17 \mathrm{~Hz}$ for $A_{\circ}=0.2 \mathrm{~g}, 16.5 \mathrm{~Hz}$ for $A_{\circ}=0.3 \mathrm{~g}$, and $15 \mathrm{~Hz}$ for $A_{\circ}=0.4 \mathrm{~g}$. These peaks appear because the mass oscillations are interrupted as they grow when the flying mass impacts the upper spring. As a result, the seismic mass displacement saturates as it covers most of the available range of motion between the upper and lower springs.

\section{Analytically-obtained Frequency Response Curves}

The frequency response curves of the VEH model are obtained using the shooting method. The shooting method is a very useful tool for finding stable and unstable periodic orbits of nonlinear dynamical systems. The method uses a series of initial guesses for the desired period and trajectory. Because the initial guesses are chosen randomly, they are corrected after each iteration using a Newton-Raphson scheme until the error is within a predefined tolerance [10]. The stability of periodic orbits, when they exist, is determined by the Floquet multipliers. If the Floquet multipliers are inside the unit circle the the orbit is stable, and if they are outside the unit circle the orbit is unstable.

The equation of motion of the $\mathrm{VEH}$ given by equation 2 is written as a system of first order differential equation as follows:

$$
\left\{\begin{array}{l}
X_{1}^{\prime}(t)=X_{2}(t) \\
X_{2}^{\prime}(t)=-\frac{X_{2}(t)\left(c_{e}+c_{m}\right)}{m}-\frac{\mathrm{F} 1}{m}-y(t)-9.81
\end{array}\right.
$$

In these approaches, the initial-value problem is converted into a two-point boundary-value problem, and the resulting system of equation is given by equation 13

$$
\left\{\begin{array}{l}
X_{1}^{\prime}(t)=X_{2}(t) \\
X_{2}^{\prime}(t)=-\frac{X_{2}(t)\left(c_{e}+c_{m}\right)}{m}-\frac{\mathrm{F} 1}{m}-y(t)-9.81 \\
X_{3}^{\prime}=\frac{d}{d t}\left(\frac{\partial X_{1}}{\partial \eta_{1}}\right)=\frac{\partial X_{2}}{\partial \eta_{1}} \\
X_{4}^{\prime}=\frac{d}{d t}\left(\frac{\partial X_{1}}{\partial \eta_{2}}\right)=\frac{\partial X_{2}}{\partial \eta_{2}} \\
X_{5}^{\prime}=\frac{d}{d t}\left(\frac{\partial X_{2}}{\partial \eta_{1}}\right)=-\left(\frac{c_{e}+c_{m}}{m}\right) \frac{\partial X_{2}}{\partial \eta_{1}}-\frac{1}{m} \frac{\partial F 1}{\partial \eta_{1}} \\
X_{6}^{\prime}=\frac{d}{d t}\left(\frac{\partial X_{2}}{\partial \eta_{2}}\right)=-\left(\frac{c_{e}+c_{m}}{m}\right) \frac{\partial X_{2}}{\partial \eta_{2}}-\frac{1}{m} \frac{\partial F 1}{\partial \eta_{2}}
\end{array}\right.
$$

Initial guess values are needed to solve the system given by equation 13 , and one way to choose such guesses is to solve the initial problem 12 by long time integration and then pick the guesses from the obtained orbit.

In the following subsections we will use the shooting model given by equation 12 to determine the response of the VEH at different amplitudes of accelerations and frequencies. We will only analyze large excitations and determine their frequency response curves and stability.

\subsection{Large Excitations}

To obtain the stable and unstable branches for large excitations we set the amplitude of acceleration to $A_{0}=0.5 \mathrm{~g}$ and solved the system of equation 13 over a range of frequencies $\omega=10 . .40 \mathrm{~Hz}$. The results of the frequency sweep using the shooting method are shown in Figure 8.

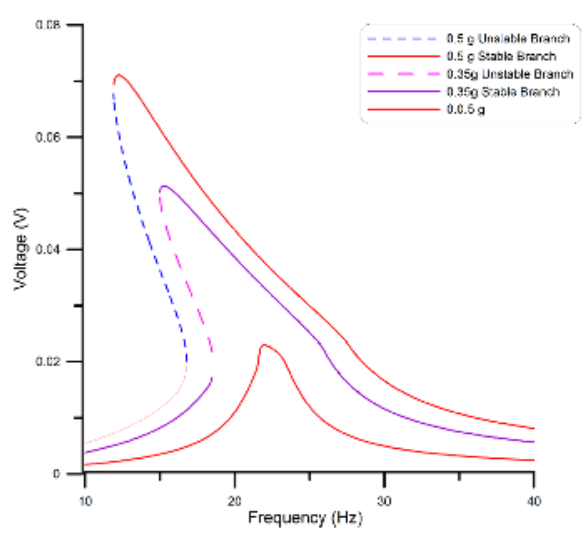

Fig. 8. Stable and Unstable Branches of the VEHs: $A_{\circ}=$ $0.5,0.35,0.05 \mathrm{~g}$ and $\omega=10 . .40 \mathrm{~Hz}$

Figure 8 shows the frequency response of the VEHs model given by the system of equation 13 for small and large amplitudes of acceleration $(0.05 \mathrm{~g}$ and $0.5 \mathrm{~g})$. We note that at low amplitudes $(0.05 \mathrm{~g})$ the system behaves close a linear spring-mass-damper and the response is maximum at a frequency in the range of its natural frequency. This results is expected since the $\mathrm{VEHs}$ is operating in a region where the mass of the oscillator remains in contact with the lower spring. As the amplitude is increased, the center frequency 
shifts to the left indicating a softening type nonlinearity. We also notice the existence of stable and unstable branches (stable branches are shown in red and the unstable branch is in blue as shown in Figure 8, this is mainly due to the fact that the mass of the oscillator looses contact with the lower spring and enters the nonlinear regions. For moderately large amplitudes $\left(0.35 \mathrm{~g} \leq A_{0} \leq 0.5 \mathrm{~g}\right)$ the system loses contact with the lower spring but does not reach the upper spring, in this region the mass $m$ compresses the lower spring fully thus invoking the higher stiffness and the nonlinear term $\alpha$ as in equation 4 . As the amplitude is increased above $(0.5 \mathrm{~g})$ the mass loses contact with the lower spring and also makes contact with the upper spring. We also note that the linear results (low accelerations) represent the solution in a narrow band around the center frequency. In the case of high amplitudes of accelerations we notice the effect of the nonlinearity as the curves are bent to the left. The stability of the periodic solutions is determined by the Floquet multipliers of the system shown in Figure 9. The figure shows the Floquet multipliers corresponding to the stable orbits since they are inside the unit circle.

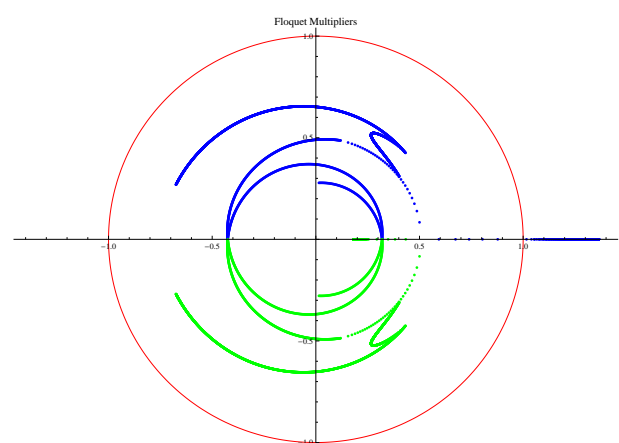

Fig. 9. VEHs Floquet Multipliers $A_{\circ}=0.4 \mathrm{~g}$ and $\omega=$ $10 . .20 \mathrm{~Hz}$

\section{Conclusion}

In this paper we modeled and analyzed the response of a vertically-aligned low-frequency springless vibration energy harvester. We tested the VEH experimentally and found that its response contains three distinct regions of operation depending on the amplitude and frequency of base excitations. In all three regions, we observed a consistent bending of the frequencyresponse curves of the coil RMS voltage to the left indicating an effective softening-type nonlinearity at high and intermediate amplitudes of acceleration. The bending of the frequency response corves indicates the presence cubic nonlinearities in the system response due to the end limiters configuration. Further, the spring-type end-limiters were found to saturate the realizable output voltage to a maximum proportional to the track length but allow for a much wider energy harvesting bandwidth as the base excitations amplitude increases.

\section{References}

1. Mostafa S. Soliman, Eihab Abdel-Rahman, E. ElSaadany, R. R. Mansour, "A wideband vibrationbased energy harvester", Journal of Micromechanics and Microengineering, Vol. 18, 2008, pp 1257-1265

2. Paul D. Mitcheson, E. M. Yeatman, "Architectures for Vibration-Driven Micropower generators", Journal of Microelectromechanical Systems, Vol. 13, 2004.

3. www.necel.com/news/en/archive/0912/0201.html

4. C. B. Williams and R. B. Yates, "Analysis of a micro-electric generator for Microsystems", in Proc. Solid-State Sensors and Actuators, 1995 and Eurosensors IX. Transducers '95, Vol. 1, 1995, pp. 369372 .

5. S. P. Beepy and T. O'Donnell, Chapter 5, pp. 130132, in Energy Harvesting Technology, Eds. S Priya and Daniel J. Inman, Springer, 2009.

6. M. A. E. Mahmoud, E. M. Abdel-Rahman, R. R. Mansour, and E. F. El-Saadany, "Springless Vibration Energy Harvesters", ASME IDETC 2010, Montreal, Canada, August 2010, DETC2010-29046.

7. Zdenek Hadas, Martin Kluge, Vladislav Singule, Cestmir Ondrusek, "Electromagnetic Vibration Power Generator" IEEE 2007.

8. N. Fondevilla, C. Serre, M. Acero, J. Esteve, and J. Morante, "Electromagnetic Harvester Device for Scavenging Ambient Mechanical Energy With Slow, Variable, and Randomness Nature", PowerMEMS 2009, Washington DC, USA, December 2009, pp. $225-228$.

9. A.H. Nayfah and D. T. Mook, Nonlinear Oscillators, John Wiley, 1997.

10. A.H. Nayfah and B. Balachandran, Applied Nonlinear Dynamics: Analytical, Computational, and Experimental Methods, John Wiley, 1997.

11. R.J.M. Vullers, R. van Schaijk, I. Doms, C. Van Hoof, R. and Mertens, "Micropower energy harvesting", Jornal of Solid-State Electronics, Vol. 53, Year 2009, pp. 684-693. 